\title{
Multiscales and cascading in isotropic turbulence
}

\author{
RAN Zheng \\ Shanghai Institute of Applied Mathematics and Mechanics, Shanghai University, Shanghai 200072, China
}

Received May 2, 2011; accepted June 14, 2011

\begin{abstract}
The explicit mapping method is used to analyze the nonlinear dynamical behavior for cascade in isotropic turbulence. This deductive scale analysis is shown to provide the first visual evidence of the celebrated Richardson-Kolmogorv cascade, and reveals in particular its multiscale character based on the statistical solutions of Navier-Stokes equations. The results also indicate that the energy cascading process has remarkable similarities with the deterministic construction rules of the logistic map. Cascade of period-doubling bifurcations have been seen in this isotropic turbulent system that exhibit chaotic behavior. The "cascade" appears as an infinite sequence of period-doubling bifurcations.
\end{abstract}

isotropic turbulence, multiscales, cascade, nonlinear bifurcation

Citation: Ran Z. Multiscales and cascading in isotropic turbulence. Chinese Sci Bull, 2011, 56: 2889-2892, doi: 10.1007/s11434-011-4675-9

The cascade picture of turbulent flows takes its origin from Richardson [1]: ...we find that convectional motions are hindered by the formation of small eddies resembling those due to dynamical instability. Although Richardson made no further use of this cascade picture at any time, Richardson cascade has played a very important role in the history of the development of turbulence. The first concept in Richardson's view of energy cascading is that the turbulence can be considered to be composed of eddies of different sizes. His notion is that the large eddies are unstable and break up, transferring their energy to somewhat smaller eddies. These smaller eddies undergo a similar break-up process, and transfer their energy to yet smaller eddies. This energy cascade-in which energy is transferred into successively smaller and smaller eddies-continues until the Reynolds number is sufficiently small that eddy motion is stable, and molecular viscosity is effective in dissipating the kinetic energy. Kolmogorov [2] added to and quantified this picture that forms the basis for nearly all work on the statistical theory of turbulence. The cascade picture is based on the intuitive notion that turbulent flows possess a hierarchical structure consisting of 'eddies' (such as Richardson's whirls

email: zran@staff.shu.edu.cn and Kolmogorov's pulsations) as a result of successive instabilities. The essence of cascade picture is in its successive hierarchical process. The general pattern of turbulent motion also can be described in the following way. The mean flow is accompanied by turbulent fluctuations imposed on it and having different scales, beginning with maximal scales of the order of the 'external scale- $L$ ' of turbulence to the smallest scales of the order of the distance $\eta$ at which the effect of viscosity becomes appreciable (the internal scale of turbulence). Most large scale fluctuations receive energy from the mean flow and transfer it to fluctuations of smaller scales. Thus, there appears to be a flux of energy transferred continuously from fluctuations of large scales to those of smaller scales. Dissipation of energy, that is, transformation of energy into heat, occurs mainly in fluctuations of scale $\eta$. The amount of energy $\varepsilon$ dissipated in unit time per unit volume is the basic characteristic of turbulent motion for all scales. Richardson's hypothesis says that, in a turbulent flow, energy is continually passed down from the large-scale structures to small scales, where it is destroyed by viscous stresses. Moreover, this is a multistage process involving a hierarchy of vortex sizes. In contrast, Kolmogorov's theory asserts that the statistical properties of the small scales depend only on the rate at which 
energy is passed down the energy cascade, and most embodiments of Kolmogorov's idea of turbulent cascade rest on heuristic modeling rather than a mathematical treatment of the Navier-Stokes equations. To date, it appears that these statements cannot be formally 'proven' in any deductive way [3-6].

In this work, we will re-examine Richardson's idea of an energy cascade, in which, it is claimed, energy is passed down from large to small scales by a repeated sequence of discrete steps. We shall show that Richardson's energy cascade is a direct consequence of vortex period-doubling bifurcation, based on the new scaling equation previously found.

For complete self-preserving isotropic turbulence [7-9], the corresponding scale equation for the length scale $l(t)$ takes the form [10-12]:

$$
\frac{\mathrm{d}^{2} l}{\mathrm{~d} t^{2}}+\frac{\left(2 a_{1}+a_{2}\right) v}{2 l^{2}} \frac{\mathrm{d} l}{\mathrm{~d} t}-\frac{a_{1} a_{2} v^{2}}{2 l^{3}}=0 .
$$

We can see that this is a closed non-linear equation, although here we are only interested in the special solutions in the following form:

$$
l(t)=l_{0}\left(t+t_{0}\right)^{\frac{1}{2}}
$$

where $l_{0}$ are parameters to be chosen [10-12]. We also know that the solutions of the turbulence scaling equation can be classified into two different kinds: P-model and $\mathrm{N}$-model, and their solutions read as

$$
\begin{gathered}
l_{\mathrm{P}}=\sqrt{2 a_{1} v\left(t+t_{0}\right)}, \\
l_{\mathrm{N}}=\sqrt{a_{2} v\left(t+t_{0}\right)} .
\end{gathered}
$$

From the small scales dynamics theory based on our recent work [12], the parameter and its variable range are given as

$$
\begin{gathered}
\sigma=\frac{a_{2}}{2 a_{1}}, \\
\sigma_{m}=\frac{2}{3}+m, \quad m=0,1,2, \cdots M+1 .
\end{gathered}
$$

A useful dimensional system must be comprised of a number of fundamental (base) entries (dimensions) that are sufficient to define the magnitude of any numerically-expressible quantity. These fundamental dimensions may be chosen rather arbitrarily, but for practical reasons, should be chosen appropriately. The selection of a dimensional system must be performed in two steps. The first step is to select the number of fundamental dimensions, and the second is to select the standard magnitude for these dimensions. For a turbulent scale system, if we choose the fundamental scale as

$$
l_{\text {ref }}=l_{\mathrm{P}} \equiv \sqrt{a_{2} v\left(t+t_{0}\right)}
$$

this analysis leads to a natural description of the cascade which is a multistage process, involving a hierarchy of vortices of varying size. Here, it is conventional to talk of these different size structures as eddies, which conjures up a picture of spherical-like objects of different diameters, denoted by $\left\{l_{m}, m=0,1, \ldots M+1\right\}$, and

$$
l_{m}=\sqrt{\frac{3}{2+3 m}} \cdot l_{\mathrm{ref}} .
$$

The $l_{m}$ represents a hierarchy of eddy sizes from the integral scale $L$ down to $\eta$. It should be noted that for all $m, l_{m}$ is a decreasing function on $m$. Then the order in volume from one generation to next is

$$
L=l_{0}>l_{1}>l_{2} \ldots>l_{m-1}>l_{m}>l_{m+1}>\ldots . . l_{M}>l_{M+1}=\eta .
$$

Let $F_{m}$ be defined as

$$
F_{m}=\left(\frac{l_{\text {ref }}}{l_{m}}\right)^{2} .
$$

It follows from the above analysis that, for each $m=0,1$, $2, \ldots M, M+1$, straightforward manipulation on $F_{m}$ defined by eq. (10) shows that $F_{m}$ are necessarily linked by a simple recursion relation:

$$
F_{m-1}-2 F_{m}+F_{m+1}=0 .
$$

This relation is valid for any 'scaling system'. However, for the present discussion on turbulent cascade, the whole solution of $F_{m}$ must satisfy two boundary conditions, where $F_{0}$ and $F_{M+1}$ are fixed according to the outer and inner turbulent scales respectively. These two boundary conditions uniquely determine the solution, and the final expression of $F_{m}$ is then

$$
F_{m}=\frac{M+1-m}{M+1} \cdot F_{0}+\frac{m}{M+1} \cdot F_{M+1} .
$$

It is natural to ask what their interpretation is in the present framework for $l_{m}$ and $l_{m+1}$. We would like to argue that eq. (12) can be rewritten in the symbolic form:

$$
l_{m+1}^{2}=\frac{l_{m}^{2}}{1+A \cdot l_{m}^{2}},
$$

where

$$
A \equiv \frac{1}{M+1}\left[\frac{1}{\eta^{2}}-\frac{1}{L^{2}}\right] .
$$

Accordingly, if the fundamental scale is chosen as

$$
l_{R}=\sqrt{2 a_{1} v\left(t+t_{0}\right)},
$$

then we have another expression for the scale

$$
q_{m+1}^{2}=q_{m}^{2}+B,
$$


where

$$
B \equiv \frac{1}{M+1} \cdot L^{2}-\frac{1}{M+1} \cdot \eta^{2}
$$

At this stage, we obtained two different expressions for the turbulent scales. Physically, the true values of the turbulent scale area will be the arithmetic mean of these two different scale areas. Here, we have

$$
y_{m} \equiv \frac{l_{m}^{2}+q_{m}^{2}}{2} .
$$

We can convert this into a difference equation only for $y_{m}$, in view of the assumption of $A<<1$ :

$$
y_{m+1}=-2 A y_{m}^{2}+y_{m}+\frac{1}{2} B .
$$

The map (19) is an instance of the logistic map [13]. Here, we could as well call it the logistic map for isotropic turbulence. On further investigation, we would not in fact use the original form of eq. (19). Moreover, we would like to use the standard form used in chaos theory $[13,14]$. This is obtained with the aid of the topological conjugate (Appendix online)

$$
x_{m+1}=a x_{m}\left(1-x_{m}\right) .
$$

It is easy to show that

$$
a=1+\sqrt{1-4 c}
$$

and

$$
c=-2+\frac{9}{2} \cdot\left(\frac{R}{R_{c}}\right)^{-\frac{3}{2}}-\frac{9}{4} \cdot\left(\frac{R}{R_{c}}\right)^{-3} .
$$

Note that the control parameter $a$ only depends on the relative Reynolds number. The conjugate $x_{m}$ can be regarded as the mean area of the Taylor micro-scale in isotropic turbulence.

Computer simulations turn out to be rich with marvelous scientific discoveries. We want to explore the behavior of the quadratic iterate (20) for all values of the parameter $a$ between 1 and 4 . Figure 1 shows the results for all parameter values $a$. This image is commonly called the Feigenbaum diagram. We note that for parameter $a>3$ the final state is not a mere point but a collection of 2,4 or more points, i.e. the well-known period-doubling. For $a=4$, of course we recover chaotic dynamic, and the points of the final state densely fill up the complete interval. Indeed, this diagram exhibits remarkable fractal behavior, and later we will see that it is closely related to the famous Mandelbrot set [14].

One essential structure seen in the Feigenbaum diagram above is that of a branching tree, which portrays the qualitative changes in the dynamical behavior of the iterate (20).

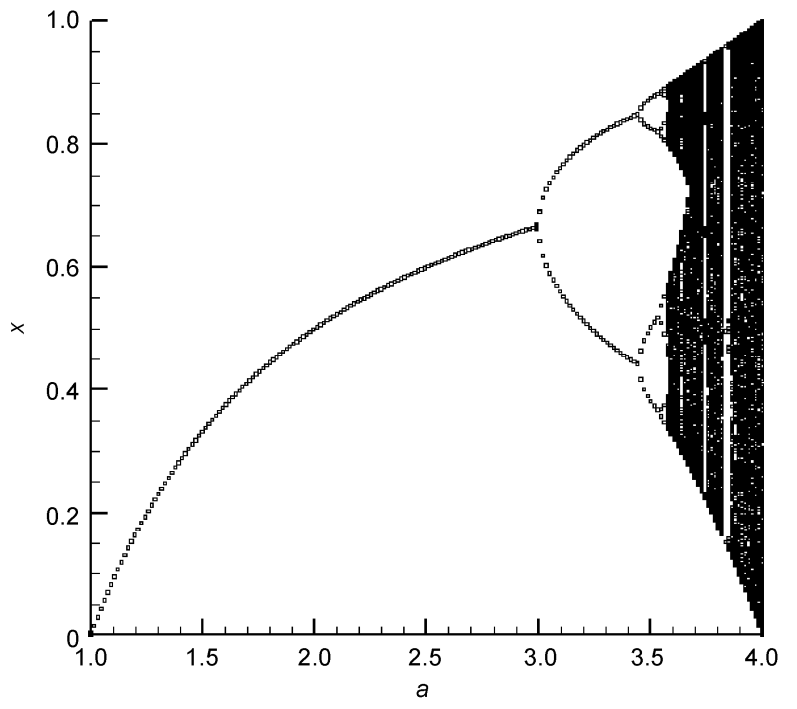

Figure 1 Feigenbaum diagram of isotropic turbulence.

The main stem bifurcates into two branches, each in turn bifurcates into two more branches, and so on. This scenario is called the period-doubling regime. The Feigenbaum diagram has self-similarity features that are both qualitative and quantitative in nature. The qualitative features are best analyzed through the methodology of fractal geometry involving self-similarity that underlies the route from order to chaos with infinite detail and complexity. This covers the main features of the classical turbulence cascade picture due to Richardson.

Eq. (20) is also well-known as the logistic equation. The equation was originally proposed for the description of the dynamics of a population of organisms that appear in discrete generations, such as insects. May [15] presented a number of examples ranging from genetics to sociology which are modeled by this equation. It underlies one of the most often discussed prototypes used in describing the complex behavior of deterministic systems. The obvious visual characteristic is the sequence of successive period doublings. It seems that as $a$ increases periodic points first appear in the order $1,2,4,8, \ldots 2^{n}, \ldots$ Specifically, let $R_{n} / R_{c}$ denote the value of bifurcation where a $2^{n}$-cycle first appears. Then Table 1 lists the critical bifurcation values. Note

Table 1 Critical Reynolds number for period-doubling bifurcation

\begin{tabular}{ccc}
\hline$n$ & & $R_{n} / R_{c}$ \\
\hline 1 & $1 \rightarrow 2$ & 2.080083823 \\
2 & $2 \rightarrow 2^{2}$ & 3.096733934 \\
3 & $2^{2} \rightarrow 2^{3}$ & 3.511518701 \\
4 & $2^{3} \rightarrow 2^{4}$ & 3.619876484 \\
5 & $2^{4} \rightarrow 2^{5}$ & 3.644190644 \\
6 & $2^{5} \rightarrow 2^{6}$ & 3.649451759 \\
$\ldots$ & $\ldots$ & $\ldots$ \\
$\infty$ & $2^{\infty} \rightarrow$ chaos chaos & 3.650890633 \\
\hline
\end{tabular}


that successive bifurcations are generated ever faster. Ultimately the bifurcation parameter converges to a limiting value $a_{\infty}$. For $a>a_{\infty}$, the orbit diagram reveals an unexpected mixture of order and chaos, with periodic windows interspersed between chaotic clouds of many periodic points. This signifies the transition from laminar flow to turbulent flow.

One of the main goals in the development of the theory of chaotic dynamical system is to make progress in understanding turbulence. The attempts to relate turbulence to chaotic motion has received strong impetus from the celebrated papers by Landau [16], Lorenz [17], and Ruelle and Takens [18]. Considerable success has been achieved mainly at the onset of turbulence. For fully developed turbulence, many questions remain unanswered. The aim of this work was to show that there are dynamical systems that are much simpler than the Navier-Stokes equations, but that can still have turbulent states and for which many concepts developed in the theory of dynamical systems can be successfully applied. In this connection we advocate a broader use of the universal properties of a wide range of isotropic turbulence phenomena [19-23].

The work was supported by the National Natural Science Foundation of China (90816013 and 10572083) and the State Key Laboratory for Complexity and Turbulence Research.
1 Richardson L F. Weather Prediction by Numerical Process. Cambridge: Cambridge University Press, 1922

2 Kolmogorov A N. Dokl Akad Nauk SSSR, 1941, 30: 9-13

3 Frisch U. Turbulence: The Legacy of A.N. Kolmogorov. Cambridge: Cambridge University Press, 1995

4 Monin A S, Yaglom A M. Statistical Fluid Mechanics: Mechanics of Turbulence. Cambridge, MA: MIT Press, 1975

5 Davidson P A. Turbulence: An Introduction for Scientists and Engineers. Oxford: Oxford University Press, 2004

6 Tsinober A. An Informal Conceptual Introduction to Turbulence. Dordrecht: Kluwer, 2001

7 Korneyev V, Sedov L I. Fluid Mech Sov Res, 1976, 5: 37-48

Sedov L I. Dokl Akad Nauk SSSR, 1944, 42: 116-119

9 Sedov L I. Similarity and Dimensional Methods in Mechanics. Moscow: Mir Publishers, 1982

10 Ran Z. Chin Phys Lett, 2008, 25: 4318-4320

11 Ran Z. Adv Appl Fluid Mech, 2009, 5: 41-67

12 Ran Z. arXiv: 0904.2036v1 [physics.flu-dyn], 2009

13 Devaney R L. An Introduction to Chaotic Dynamical Systems. Reading, MA: Addison-Wesley, 1987

14 Schuster H G. Deterministic Chaos: An Introduction. 2nd ed. New York: VCH, New York, 1988

15 May R M. Nature, 1976, 26: 459-467

16 Landau L D. Dokl Akad Naul SSSR, 1944, 44: 311-314

17 Lorenz E N. J Atmos Sci, 1963, 20: 130-141

18 Ruelle D, Takens F. Commun Math Phys, 1971, 20: 167-192

19 Meneveau C, Sreenivasan K R. J Fluid Mech, 1991, 224: 429-484

20 She Z S, Leveque E. Phys Rev Lett, 1994, 72: 336-339

21 Sun H G, Chen W. Sci China Ser E-Tech Sci, 2009, 52: 680-683

22 Zheng X J, Zhang J H. Chinese Sci Bull, 2010, 55: 3107-3112

23 Lv Y, Wang Z H, Pei H, et al. Chinese Sci Bull, 2010, 55: 1231-1239

Open Access This article is distributed under the terms of the Creative Commons Attribution License which permits any use, distribution, and reproduction in any medium, provided the original author(s) and source are credited.

\section{Supporting Information}

Appendix: Equivalence of quadratic polynomials on topological conjugacy

The supporting information is available online at csb.scichina.com and www.springerlink.com. The supporting materials are published as submitted, without typesetting or editing. The responsibility for scientific accuracy and content remains entirely with the authors. 